\title{
APPROXIMATE EIGENFUNCTIONS OF THE LAPLACIAN
}

\author{
J. V. RALSTON
}

\section{Introduction}

Let $M$ be a compact orientable $(n+1)$-dimensional riemannian manifold, and let $\Gamma$ be a closed geodesic on $M$. We say $\Gamma$ is stable if the Poincare map associated with $\Gamma$ (this is defined in $\S 1$ ) splits into a direct sum of rotations through distinct angles $\theta_{1}, \cdots, \theta_{n}, 0<\theta_{i}<2 \pi, \theta_{i} \neq 2 \pi-\theta_{j}$ for all $i, j$. Let $\Delta$ denote the Laplace-Beltrami operator on $M$. Guillemin and Weinstein [5] have recently proved the following.

Theorem I. If there is a stable closed geodesic on $M$ of length $L$, then, given any multi-index $\alpha$, there are at least two eigenvalues $\lambda_{m}$ of $\Delta$, counted by multiplicity, satisfying $\sqrt{-\lambda_{m}}=k_{m}+0\left(m^{-\frac{1}{2}}\right)$, where $k_{m}=L^{-1}(2 \pi m+$ $\left.\left(\alpha_{1}+\frac{1}{2}\right) \theta_{1}+\cdots+\left(\alpha_{n}+\frac{1}{2}\right) \theta_{n}+\pi p_{0}\right)$. Here $p_{0}=0$ or 1 and is determined by the behavior of the Jacobi fields along $\Gamma$.

Since a rotation through an angle $\theta$ can turn into a rotation through $2 \pi-\theta$ if one changes bases, there is a technical condition that determines which of these rotations one chooses in selecting $\theta_{1}, \cdots, \theta_{n}$ in Theorem $\mathrm{I}$. We omit this here; see $\S 2$.

Guillemin and Weinstein's proof of Theorem I is based on the construction of an isometry from $L^{2}\left(S^{1}\right)$ to $L^{2}(M)$ that approximately intertwines $d^{2} / d \theta^{2}$ and $\Delta$. The isometry is a Fourier integral operator of a new type developed by Guillemin in [4].

Our objective here is to prove Theorem I and its analogue for nonorientable $M$ by constructing approximate solutions $u_{m}$ to the equations $\left(\Delta+k_{m}^{2}\right) u=0$. The functions $u_{m}$ are probably very close to the image of $\left\{e^{i m \theta}\right\}_{m=1}^{\infty}$ under the isometry used by Guillemin and Weinstein. However, we construct them by beginning with the ansatz of geometrical optics and using a complex phase function $\psi$ with $\operatorname{Im} \psi>0$ off $\Gamma$ and $\operatorname{Im} \psi=0$ on $\Gamma$. The resulting $u_{m}$ are very small outside a tube around $\Gamma$ with radius $0\left(m^{-\frac{1}{2}}\right)$. The construction is quite explicit, expressing the $u_{m}$ in terms of the Jacobi fields along $\Gamma$.

Our approach is derived from the work of Babich and Lazutkin [2] who used a similar method to prove Theorem $I$ in the case $n=1$. We found the idea which enabled us to carry out the construction for general $n$ in the paper [6] of Hörmander.

Received March 22, 1975. 
In $\S 4$ we sketch the extension of these results to the case of a closed piecewise geodesic arc $\Gamma$ reflected off $\partial M$ at its corners. In the case that $\Gamma$ consists of two copies of a single arc invariant under reflection in $\partial M$, the functions $u_{m}$ are known as "bouncing ball waves". Such waves were discussed by Keller and Rubinow in a paper [8] which' introduced the idea that closed ray paths could be used to predict eigenvalues of the Laplacian. As in the case of a closed geodesic, when $n=1$ the results in $\S 4$ for bouncing ball waves are due to Lazutkin [9] and also Smith [10].

The significance of the approximate eigenfunctions $u_{m}$ is not clear. In [1] Arnol'd has given an example (in the case of bouncing ball waves with $n=1$ ) where they approximated no true eigenfunctions of $\Delta$. In $\S 5$ we point out that in all cases (even when $M$ is not compact) the $u_{m}$ for $m$ large are the amplitudes of very long-lasting standing wave vibrations of $M$. This observation also appears in [1].

The author is indebted to Professor Alan Weinstein for many helpful discussions of this work. ${ }^{1}$

\section{Description of method}

We will look for approximate solutions to $\left(\Delta+k^{2}\right) u=0$, which have the form

$$
u=e^{i k \psi(x)}\left(a_{0}(x)+a_{1}(x) / k+\cdots+a_{N}(x) / k^{N}\right) .
$$

When $\psi$ is real-valued, (1.1) is the standard ansatz of geometrical optics. The only novelty here is that we allow $\operatorname{Im} \psi \geq 0$.

In local coordinates the principal symbol of $\Delta$ has the form

$$
p(x, \xi)=\sum g^{i j}(x) \xi_{i} \xi_{j},
$$

where the line element $d s^{2}=\sum g_{i j} d x_{i} d x_{j}$, and $g^{i j}=\left(g_{i j}\right)^{-1}$. When $u$ has the form (1.1), the coefficient $c_{s}$ of $k^{2-s} e^{i k \psi}$ in $\left(\Delta+k^{2}\right) u$ is given by

$$
\begin{aligned}
& c_{0}=\underline{c}_{0} a_{0}=(1-p(x, d \psi)) a_{0}, \\
& c_{1}=i\left(\frac{\partial p}{\partial \xi}(x, d \psi) \cdot \frac{\partial a_{0}}{\partial x}+(\Delta \psi) a_{0}\right)+\underline{c}_{0} a_{1}, \\
& \left.c_{s}=i\left(\frac{\partial p}{\partial \xi}(x, d \psi) \cdot \frac{\partial a_{s-1}}{\partial x}+(\Delta \psi) a_{s-1}-i \Delta a_{s-2}\right)\right)+\underline{c}_{0} a_{s},
\end{aligned}
$$

where $a_{-2}=a_{-1}=a_{N+1}=a_{N+2} \equiv 0$. To solve $\underline{c}_{0} \equiv 0$ one prescribes $\psi$ and its normal derivative on a surface $S$ transverse $\Gamma$ so that $\underline{c}_{0}=0$ on $S$. Then one solves the characteristic equations $\dot{x}=\partial p / \partial \xi, \dot{\xi}=-\partial p / \partial x$ with $x(0) \in S$, $\xi(0)=d \psi(x(0))$. If one prescribes complex values for $\psi$ on $S$, it is clear that

\footnotetext{
${ }^{1}$ Added in proof. For additional results and references see the author's article, Comm.
} Math. Phys. 51 (1976) 219-242. 
the coordinates $x$ must be complex, and the characteristic equations make no sense, since the metric $g^{i j}$ is not necessarily analytic in $x$. Hence we will not attempt to solve $\underline{c}_{0} \equiv 0$, but instead will try to make $\underline{c}_{0}=0$ to third order along $\Gamma$. To do this we will use an idea which we believe is due to Hörmander (see the remarks following Theorem 3.4.1 of [6]).

If $\psi$ were real and one solved $\underline{c}_{0} \equiv 0$, the submanifold $\Lambda \subset T^{*}(M)$ given by $\left\{\left(x, d_{x} \psi\right)\right\}$ would be invariant under the characteristic flow. Moreover, the conic submanifold $C=\left\{\left(x, c d_{x} \psi\right): x \in \Gamma, c \in \boldsymbol{R}_{+}\right\}$would be invariant under the characteristic flow, because $\Gamma$ is a geodesic. Let $\sigma$ denote the symplectic 2 -formin local coordinates

$$
\sigma=\sum d \xi_{i} \wedge d x_{i}
$$

We define, for $p \in \gamma=\left\{\left(x, d_{x} \psi\right): x \in \Gamma\right\}$,

$$
\begin{aligned}
& J_{p}=\left\{v \in T_{p}\left(T^{*}(M)\right): \sigma(v, t)=0, t \in T_{p}(C)\right\} \\
& L_{p}=\left\{v \in T_{p}(\Lambda): \sigma(v, t)=0, t \in T_{p}(C)\right\}
\end{aligned}
$$

and let $\Phi$ denote the flow on $\left\{T_{p}\left(T^{*}(M)\right): p \in \gamma\right\}$ induced by the characteristic flow on $T^{*}(M)$. Since $\Phi$ preserves $\sigma$ and leaves $\left\{T_{p}(C): p \in \gamma\right\}$ invariant, $\Phi$ leaves $\left\{J_{p}: p \in \gamma\right\}$ invariant. Moreover, if $\Lambda$ is invariant under the characteristic flow, then $\Phi$ must leave $\left\{L_{p}: p \in \gamma\right\}$ invariant.

If we introduce coordinates $x_{0}, x_{1}, \cdots, x_{n}$ on $M$ with $x^{\prime}=\left(x_{1}, \cdots, x_{n}\right)$ vanishing on $\Gamma$ and $\partial / \partial x_{i}, i=1, \cdots, n$, perpendicular to $\partial / \partial x_{0}$ along $\Gamma$, then

$$
L_{p}=\left\{\left(\delta x^{\prime}, \delta \xi^{\prime}\right): \delta \xi^{\prime}=d_{x^{\prime}}^{2} \psi \delta x^{\prime}\right\}
$$

Hence, knowing $L_{p}, p \in \gamma$, is equivalent to knowing $d_{x^{\prime}}^{2} \psi$ on $\Gamma$.

Thus to solve $\underline{c}_{0}=0$ to third order along $\Gamma$ we first choose $\psi$ real-valued on $\Gamma$ so that $\underline{c}_{0}=0$ on $\Gamma$ (there are two possible choices of $d \psi$ here - leading to $\psi_{+}$and $\left.\psi_{-}\right)$. Then we pick a complex $n$-dimensional isotropic subspace $L_{p_{0}}$ of the complexification of $J_{p_{0}}$, for some $p_{0} \in \gamma$, and consider its orbit under $\Phi$ (from this point on $J_{p}$ is always complexified). Assuming this orbit is periodic, i.e., there is just one subspace in it sitting over each $p \in \gamma$, and assuming the projection of this subspace onto the complexification of $T_{p}(M)$ considered as a subspace of $J_{p}$ is always nonsingular, we can determine $d_{x^{\prime}}^{2} \psi=B\left(x_{0}\right)$ along $\Gamma$. Then we define $\psi=\left.\psi\right|_{x^{\prime}=0}+\frac{1}{2} x^{\prime} \cdot B\left(x_{0}\right) x^{\prime}$. The reason for this roundabout approach is that if one simply writes down the differential equation $B\left(x_{0}\right)$ must satisfy in order that $\underline{c}_{0}=0$ on $\Gamma$ to third order, one gets a formidable nonlinear ordinary differential equation in matrices. In contrast the flow $\Phi$ arises from a linear ordinary differential equation-the differential equation satisfied by the Jacobi fields along $\Gamma$. Taking the approach of Hörmander described here, one finds it is easy to express $B\left(x_{0}\right)$ in terms of the (complex) Jacobi 
fields. $^{2}$

The Poincaré map is the map of $J_{p}$ to itself obtained by following to flow $\Phi$ once around $\Gamma$. The hypothesis that $\Gamma$ is stable means that the Poincare map has distinct eigenvalues $\lambda_{1}, \bar{\lambda}_{1}, \cdots, \lambda_{n}, \bar{\lambda}_{n}$ with $\left|\lambda_{i}\right|=1$. As will be shown in $\S 2$, this implies that for each of the two choices of $d \psi$ on $\Gamma$ there is a unique choice of $L_{p_{0}}$ such that the orbit of $L_{p_{0}}$ is periodic and $\operatorname{Im} B\left(x_{0}\right)$ is positive definite for $x_{0} \in \Gamma$. Thus we can construct two phase functions $\psi_{+}$and $\psi_{-}$, with $\operatorname{Im} \psi_{ \pm}=0$ on $\Gamma$ and $\operatorname{Im} \psi_{ \pm}>0$ off $\Gamma$, which are quadratic in $x^{\prime}$ and satisfy $\underline{c}_{0}=0$ to second order. Actually $\psi_{-}=-\bar{\psi}_{+}$and we only construct $\psi_{+}$in $\S 2$.

Let \|\| denote the $L^{2}$-norm over a fixed $k$-independent neighborhood of $\Gamma$. Since we are solving $\underline{c}_{0}=0$ only to third order on $\Gamma$, we will not attempt to solve $\left(\Delta+k^{2}\right) u=0$ more accurately than

$$
\left\|\left(\Delta+k^{2}\right) u\right\|=O\left(\left\|k^{2} \underline{c}_{0} a_{0} e^{-k \operatorname{Im} \psi}\right\|\right)=O\left(k^{-\frac{1}{1} n-\frac{1}{2}(l-1)}\right),
$$

where $a_{0}$ vanishes to order $l$ on $\Gamma$. Noting that multiplying a function of the form $f\left(x_{0}, x^{\prime}\right) e^{-k\left|x^{\prime}\right| 2}$ by a linear function in $x^{\prime}$ essentially multiplies its norm by $k^{-\frac{1}{2}}$, we see the contributions to $\left(\Delta+k^{2}\right) u$ from terms in $a_{0}$ which vanish to order $l+1$ on $\Gamma$ will be negligible. Applying this reasoning to $a_{1}, \cdots, a_{N}$ one arrives at the ansatz: $a_{s}$ is a homogeneous polynomial in $x^{\prime}$ of degree $l-2 s$ with coefficients depending on $x_{0}$, and $a_{N}$ is degree 0 or 1 in $x^{\prime}$. Then, assuming this ansatz, one solves $c_{s}=0$ to order $l-2 s+3$ on $\Gamma, s=1, \cdots, N+1$, which implies (1.2).

We solve the equations $c_{k}=0, k=1, \cdots, N+1$ to the required accuracy on $\Gamma$ in $\S 3$. The resulting coefficients $a_{0}, \cdots, a_{N}$ must be chosen so that they are multipled by the same factor $e^{-i \beta}, \beta$ real, as one goes once around $\Gamma$. However, once $a_{0}$ has been chosen so that this holds, it will hold for $a_{1}, \cdots, a_{N}$. Since $\psi_{+}$increases by the length $L$ of $\Gamma$ as one goes once around $\Gamma$, the functions

$$
u_{m}=e^{i k_{m} \psi+}\left(a_{0}+a_{1} / k+\cdots+a_{N} / k^{N}\right),
$$

where $k_{m}=(2 \pi m+\beta) / L, m \in Z_{+}$will be well-defined near $\Gamma$.

\section{Construction of the phase function}

In this section and the next we will use the following coordinate system $\left(x_{0}, x^{\prime}\right)$ near $\Gamma$. At a point $p_{0}$ on $\Gamma$ choose an orthonormal frame $v_{1}\left(p_{0}\right), \cdots$, $v_{n}\left(p_{0}\right)$ orthogonal to $\Gamma$ and, using parallel transport along $\Gamma$, extend $v_{1}, \cdots, v_{n}$ to parallel vector fields along $\Gamma$. If the length of $\Gamma$ is $L$, we assign coordinates

\footnotetext{
${ }^{2} \mathrm{~N}$. Grossman has pointed out that the "formidable nonlinear ordinary differential equation" is a matrix Riccati equation and the process described here is related to the method used to reduce such equations to linear equations.
} 
$x_{0}, x_{1}, \cdots, x_{n}$ where $0 \leq x_{0}<L$ to the image of $x_{1} v_{1}(p)+\cdots+x_{n} v_{n}(p)$ under the exponential map at $p$ where $p$ is the point on $\Gamma$ at distance $x_{0}$ from $p_{0}$. Hence the orthonormal frames $\partial / \partial x_{1}, \cdots, \partial / \partial x_{n}$ on $\Gamma$ at $x_{0}=0$ and $x_{0}=L$ are related by the orthogonal holonomy matrix $O$.

In these coordinates the metric $g^{i j}$ satisfies $g^{i j}=\delta_{i j}+O\left(\left|x^{\prime}\right|\right), g^{0 j}=O\left(\left|x^{\prime}\right|^{2}\right)$ for $j \neq 0$ and $g^{00}=1+O\left(\left|x^{\prime}\right|^{2}\right)$. Moreover, if we define $\psi=x_{0}$ on $\Gamma$, the differential equation governing the flow $\Phi$ on $\left\{J_{p}: p \in \gamma\right\}$ is given by

$$
\delta \dot{x}^{\prime}=\delta \xi^{\prime}, \quad \delta \dot{\xi}^{\prime}=-\frac{1}{2} \frac{\partial^{2} g^{00}}{\partial x^{\prime} \partial x^{\prime}}\left(x_{0}, 0\right) \delta x^{\prime},
$$

where over a letter denotes differentiation with respect to $x_{0}$. To verify (2.1) we note that in these coordinates $J_{p}$ is the (complex) span of

$$
\left.\frac{\partial}{\partial x_{1}}\right|_{p}, \cdots,\left.\frac{\partial}{\partial x_{n}}\right|_{p}, \frac{\partial}{\partial \xi_{1}}\left|, \cdots, \frac{\partial}{\partial \xi_{n}}\right|_{p}
$$

Then we consider a one-parameter family of solutions of the characteristic equations $(x(t, h), \xi(t, h))$ satisfying

$$
(x(t, 0), \xi(t, 0))=(2 t, 0, \cdots, 0,1,0, \cdots, 0) .
$$

Then letting $x_{h}(t)=(\partial x / \partial h)(t, 0), \xi_{h}(t)=(\partial \xi / \partial h)(t, 0)$ and differentiating the characteristic equations we have

$$
\begin{aligned}
& \dot{x}_{h}=\frac{\partial^{2} p}{\partial \xi^{2}}(x(t, 0), \xi(t, 0)) \xi_{h}+\frac{\partial^{2} p}{\partial x \partial \xi}(x(t, 0), \xi(t, 0)) x_{h}, \\
& \dot{\xi}_{h}=-\frac{\partial^{2} p}{\partial x \partial \xi}(x(t, 0), \xi(t, 0)) \xi_{h}-\frac{\partial^{2} p}{\partial x^{2}}(x(t, 0), \xi(t, 0)) x_{h} .
\end{aligned}
$$

Using the properties of $g^{i j}$ given earlier these equations reduce to

$$
\dot{x}_{h}=2 \xi_{h}, \quad \dot{\xi}_{h}=-\frac{\partial^{2} g^{00}}{\partial x^{2}}(2 t, 0) x_{n} .
$$

Now, changing the curve parameter to $x_{0}$ and restricting to the $\left(x^{\prime}, \xi^{\prime}\right)$ components, one has (2.1).

The hypothesis that the eigenvalues of the Poincare map lie on the unit circle and are distinct implies that we can find complex solutions $f_{i}=\left(\varphi_{i}, \dot{\varphi}_{i}\right), i=$ $1, \cdots, n$, of (2.1) satisfying

$$
\left(\varphi_{i}(L), \dot{\varphi}_{i}(L)\right)=\lambda_{i}\left(O \varphi_{i}(0), O \dot{\varphi}_{i}(0)\right),
$$

where $\lambda_{1}, \bar{\lambda}_{1}, \cdots, \lambda_{n}, \bar{\lambda}_{n}$ are the eigenvalues of the Poincaré map.

Let $L\left(x_{0}\right)$ denote the complex span of $f_{1}\left(x_{0}\right), \cdots, f_{n}\left(x_{0}\right) . L\left(x_{0}\right)$ is periodic in 
the sense described in $\S 1$. To carry out the construction of a phase function from $L\left(x_{0}\right)$, we need to show $L\left(x_{0}\right)$ is isotropic and $\left\{\varphi_{1}\left(x_{0}\right), \cdots, \varphi_{n}\left(x_{0}\right)\right\}$ is linearly independent $0 \leq x_{0} \leq L$.

We have

$$
\varphi_{i} \cdot \dot{\varphi}_{j}-\dot{\varphi}_{i} \cdot \varphi_{j}=c_{i j}, \quad \varphi_{i} \cdot \dot{\bar{\varphi}}_{j}-\dot{\varphi}_{i} \cdot \bar{\varphi}_{j}=d_{i j} .
$$

where "." denotes the vector dot product, "_-" over a letter denotes conjugation, and $c_{i j}$ and $d_{i j}$ are constants. (2.3) is just the statement that $\Phi$ is a real symplectic map, but one can verify it directly using (2.1). Now (2.2) implies $c_{i j}$ $=\lambda_{i} \lambda_{j} c_{i j}$ and $d_{i j}=\lambda_{i} \bar{\lambda}_{j} d_{i j}$, and we conclude $c_{i j}=0, \forall i, j$ and $d_{i j}=0, i \neq j$. If $d_{i i}=0$ for any $i$, we have $\left(\varphi_{i}, \dot{\varphi}_{i}\right)=0$, a contradiction. Thus normalizing $\varphi_{i}$ and interchanging $\lambda_{i}$ and $\bar{\lambda}_{i}$ where necessary we can achieve $d_{i i}=-2 \sqrt{-1}$. Note that now $L\left(x_{0}\right)$ is uniquely determined.

That $c_{i j} \equiv 0$ is simply the statement that $L\left(x_{0}\right), 0 \leq x_{0} \leq L$, is isotropic. Suppose $\sum_{i=l}^{n} a_{i} \varphi_{i}\left(x_{0}\right)=0$. Then by (2.3)

$$
\begin{gathered}
\left(\sum a_{i} \dot{\varphi}_{i}\right) \cdot \bar{\varphi}_{j}=2 \sqrt{-1} a_{j}, \\
0=\sum_{j=1}^{n}\left(\sum_{i=1}^{n} a_{i} \dot{\varphi}_{i}\right) \cdot \bar{a}_{j} \bar{\varphi}_{j}=2 \sqrt{-1}\left(\sum_{j=1}^{n}\left|a_{j}\right|^{2}\right),
\end{gathered}
$$

and we have $a_{j}=0, j=1, \cdots, n$, and $\left\{\varphi_{i}\left(x_{0}\right)\right\}_{i=1}^{n}$ is linearly independent.

The results of the preceding paragraph show that the equations

$$
B \varphi_{i}=\dot{\varphi}_{i}, \quad i=1, \cdots, n,
$$

define a symmetric $n \times n$ matrix function. The argument of $\S 1$ shows the phase function

$$
\psi=x_{0}+\frac{1}{2} x^{\prime} \cdot B\left(x_{0}\right) x^{\prime}
$$

satisfies the eichonal equation $\underline{c}_{0}=0$ to third order on $\Gamma$. This can also be checked by direct computation.

For our construction it is also necessary that $\operatorname{Im} B$ be positive definite. However, we have, writing $B=C+i A$,

$$
\begin{aligned}
& \bar{\varphi}_{j} \cdot A \varphi_{i}=\frac{1}{2 \sqrt{-1}}\left(\bar{\varphi}_{j} \cdot B \varphi_{i}-\bar{B} \bar{\varphi}_{j} \cdot \varphi_{i}\right) \\
& =\frac{1}{2 \sqrt{-1}}\left(\bar{\varphi}_{j} \cdot \dot{\varphi}_{i}-\dot{\bar{\varphi}}_{j} \cdot \varphi_{i}\right)=\delta_{i j} .
\end{aligned}
$$

\section{Amplitudes and the eigenvalue condition}

Following the ansatz that $a_{s}$ is a homogeneous polynomial in $x^{\prime}$ of degree $l-2 s$, and ignoring terms vanishing on $\Gamma$ to order $l+1$, the equation $c_{1}=0$ becomes 


$$
0=2 \frac{\partial a_{0}}{\partial x_{0}}+2 B x^{\prime} \cdot \frac{\partial a_{0}}{\partial x^{\prime}}+(\operatorname{trace} B) a_{0} .
$$

In ordinary geometrical optics the equation $c_{1}=0$ (given $\underline{c}_{0}=0$ ) means that $a_{0}$, considered as a $\frac{1}{2}$-density, is invariant under the flow defined by

$$
\dot{x}=\frac{\partial p}{\partial \xi}(x, d \psi) .
$$

If we continue the approach taken in $\S 1$, we should interpret this invariance infinitesimally along $\Gamma$ to solve (3.1). To do this, we note that the $\varphi_{i}, i=1$, $\cdots, n$, are the $x^{\prime}$ components of derivatives of the flow defined by (3.2) in directions normal to $\Gamma$ and the derivative of the flow with respect to $x_{0}$ is just $(1,0, \cdots, 0)$ on $\Gamma$. Hence, if we define $Z$ to be the $n \times n$ matrix with columns $\varphi_{1}, \cdots, \varphi_{n}$ on $\Gamma$, det $Z$ is a constant multiple of the Jacobian of the flow defined by (3.2).

To check this argument one notes that $B=\left(d Z / d x_{0}\right) Z^{-1}$ and uses the identity

$$
\text { trace } \frac{d Z}{d x_{0}} Z^{-1}=\frac{d}{d x_{0}} \log \operatorname{det} Z .
$$

Defining $e_{0}=(\operatorname{det} Z)^{\frac{1}{2}} a_{0}$, it then follows that

$$
0=2 \frac{\partial e_{0}}{\partial x_{0}}+2 B x^{\prime} \cdot \frac{\partial e_{0}}{\partial x^{\prime}}
$$

as is implied by the invariance of $a_{0}$, considered as a $\frac{1}{2}$-density, under a flow whose Jacobian is a constant multiple of $\operatorname{det} Z$.

(3.4) means that the function $e_{0}$ is invariant under the flow defined by (3.2), modulo terms vanishing to order $l+1$ on $\Gamma$. Since $e_{0}$ is a homogeneous polynomial in $x^{\prime}$ and invariance under a flow is preserved under sums and products, it will suffice to solve (3.3) in the case where $e_{0}$ is homogeneous linear function of $x^{\prime}$ (with coefficients depending on $x_{0}$ ).

Let $\Lambda_{t}$ denote the flow defined by (3.2). The differential version of $e_{0} \circ \Lambda_{t}=$ $e_{0}$ is

$$
\Lambda_{t}^{*}\left(d e_{0}\right)=d e_{0} .
$$

We will only use (3.5) on $\Gamma$. Since $d e_{0}$ on $\Gamma$ annihilates vectors tangent to $\Gamma$, (3.5) simply means

$$
\frac{\partial e_{0}}{\partial x^{\prime}} \cdot \varphi_{i}=c_{i} \quad \text { on } \quad \Gamma, \quad i=1, \cdots, n,
$$

where $c_{i}$ is a constant. Hence referring to (2.4) one sees $\partial e_{0} / \partial x^{\prime}$ is in the span of $\left\{A \bar{\varphi}_{1}, \cdots, A \bar{\varphi}_{n}\right\}$. 
Turning now to the case where $e_{0}$ is homogeneous of degree $l$, the preceding paragraph implies that $e_{0}$ has the form (in multi-index notation)

$$
e_{0}=\sum_{|\alpha|=l} a_{\alpha}\left(x^{\prime} \cdot A \bar{\varphi}_{1}\right)^{\alpha_{1}} \cdots\left(x^{\prime} \cdot A \bar{\varphi}_{n}\right)^{\alpha_{n}}
$$

Each "monomial" $e_{0 \alpha}=\left(x^{\prime} \cdot A \bar{\varphi}_{1}\right)^{\alpha_{1}} \cdots\left(x^{\prime} \cdot A \bar{\varphi}_{n}\right)^{\alpha_{n}}$ in this sum satisfies $e_{0 \alpha}\left(L, O x^{\prime}\right)=\bar{\lambda}_{1}^{\alpha_{1}} \ldots \bar{\lambda}_{n}^{\alpha_{n}} e_{0, \alpha}\left(0, x^{\prime}\right)=e^{-i \beta} e_{0 \alpha}\left(0, x^{\prime}\right)$ with real $\beta$, and we will see each $e_{0 \alpha}$ leads to a distinct approximate solution to $\left(\Delta+k^{2}\right) u=0$. Thus, without loss of generality, we assume $e_{0}$ is one of these monomials. Therefore we have

$$
a_{0}=(\operatorname{det} Z)^{-\frac{1}{2}}\left(x^{\prime} \cdot A \bar{\varphi}_{1}\right)^{\alpha_{1}} \cdots\left(x^{\prime} \cdot A \bar{\varphi}_{n}\right)^{\alpha_{n}}, \quad|\alpha|=l .
$$

One may also check directly that that $a_{0}$ satisfies (3.1).

In solving $c_{s}=0$ on $\Gamma$ to order $l-2 s+3$ for $s=2, \cdots, N+1$ we will abandon the geometrical approach used to solve $\underline{c}_{0}=0$ and $c_{1}=0$. This will be done primarily because we do not know how to solve them geometrically, but also because, given our solutions to $\underline{c}_{0}=0$ and $c_{1}=0$, the rest of the equations are easy to solve.

We note first that (2.4) implies $\left(d / d x_{0}\right)\left(A \bar{\varphi}_{j}\right)+B A \bar{\varphi}_{j}=0$. Then we have

$$
\frac{d}{d x_{0}}\left(\bar{\varphi}_{i} \cdot A \bar{\varphi}_{j}\right)=\bar{B} \bar{\varphi}_{i} \cdot A \bar{\varphi}_{j}-\bar{\varphi}_{i} \cdot B A \bar{\varphi}_{j}=-2 \sqrt{-1} A \bar{\varphi}_{i} \cdot A \bar{\varphi}_{j} .
$$

Let $C$ denote the differential operator

$$
C u=\frac{\partial}{\partial x^{\prime}}\left(A^{-1} \frac{\partial u}{\partial x^{\prime}}\right) \text {. }
$$

Ignoring terms vanishing on $\Gamma$ to order $l-2 s+3$ the equation $c_{s}=0$ becomes

$$
2 \frac{\partial a_{s-1}}{\partial x_{0}}+2 B x^{\prime} \cdot \frac{\partial a_{s-1}}{\partial x^{\prime}}+(\operatorname{trace} B) a_{s-1}=\sqrt{-1}\left(\frac{\partial^{2} a_{s-2}}{\partial x_{1}^{2}}+\cdots+\frac{\partial^{2} a_{s-2}}{\partial x_{n}^{2}}\right)
$$

Then using (3.6) and the definition of $C$ one sees (3.7) will be satisfied if $a_{s}=-\frac{1}{4} C a_{s-1} / s, s=1, \cdots, N$.

To sum up we have shown that if

$$
u=e^{i k\left(x_{0}+\frac{1}{2} x^{\prime} \cdot B x^{\prime}\right)}\left(a_{0}+\frac{-1}{4 k} C a_{0}+\cdots+\frac{1}{N !}\left(\frac{-1}{4 k}\right)^{N} C^{v} a_{0}\right)
$$

where $a_{0}=(\operatorname{det} Z)^{-\frac{1}{2}}\left(x^{\prime} \cdot A \bar{\varphi}_{1}\right)^{\alpha_{1}} \cdots\left(x^{\prime} \cdot A \bar{\varphi}_{n}\right)^{\alpha_{n}}$, then $\left\|\left(\Delta+k^{2}\right) u\right\|=O\left(k^{\left.-\frac{1}{n-\frac{1}{2}(l-1)}\right)}\right.$. However, $u$ is not well-defined in a neighborhood of $\Gamma$ unless $u\left(0, x^{\prime}\right)=$ $u\left(L, O x^{\prime}\right)$. As noted in $\S 1$, this leads to the eigenvalue condition on $k$. 
To determine the relation between $u\left(0, x^{\prime}\right)$ and $u\left(L, O x^{\prime}\right)$ we note that (see 3.3)

$$
\frac{d}{d x_{0}}(\operatorname{Arg} \operatorname{det} Z)=\operatorname{trace} A
$$

and (2.4) implies trace $A>0$. Hence we may choose $p \in Z, p \geq 0$ and $\theta_{0}$, $0 \leq \theta_{0}<2 \pi$, so that

$$
\int_{0}^{L} d(\operatorname{Arg} \operatorname{det} Z)=2 \pi p+\theta_{0} .
$$

Let $\lambda_{i}=e^{\sqrt{-1} \theta_{i}}, 0<\theta_{i}<2 \pi$. We have

$$
Z(L)=O Z(0)\left(\begin{array}{lll}
\lambda_{1} & & 0 \\
& \ddots & \\
0 & & \lambda_{n}
\end{array}\right),
$$

and hence $\operatorname{det} Z(L)=\lambda_{1} \cdots \lambda_{n} \operatorname{det} Z(0) \operatorname{det} O$. Let $(-1)^{\nu}=\operatorname{det} O, \nu=0$ or 1. $\nu=0$ if a neighborhood of $\Gamma$ is orientable, $\nu=1$ if it is not. We then have

$$
\theta_{0} \equiv\left(\theta_{1}+\cdots+\theta_{n}+\pi \nu\right), \quad \bmod 2 \pi,
$$

and

$$
u\left(L, O x^{\prime}\right)=e^{-\sqrt{-}-1\left(\pi p+\theta_{0} / 2\right)} \lambda_{1}^{-\alpha_{1}} \cdots \lambda_{n}^{-\alpha_{n}} e^{\sqrt{-i} k L} u\left(0, x^{\prime}\right) .
$$

Thus, setting $\theta_{0}=\theta_{1}+\cdots+\theta_{n}+\pi \nu+2 \pi p^{\prime}$, we see $u$ is a well-defined smooth function near $\Gamma$ provided

$$
\begin{aligned}
k=k_{r, \alpha}= & \frac{1}{L}\left(2 \pi r+\left(\alpha_{1}+\frac{1}{2}\right) \theta_{1}\right. \\
& \left.+\cdots+\left(\alpha_{n}+\frac{1}{2}\right) \theta_{n}+\pi\left(p+p^{\prime}\right)+\frac{\pi}{2} \nu\right) .
\end{aligned}
$$

Finally, using the change of variables $\left(y_{0}, y^{\prime}\right)=\left(x_{0}, k^{\frac{1}{2}} x^{\prime}\right)$ one checks easily that $\left\|u_{r, \alpha}\right\| \geq c_{\alpha} k_{r, \alpha}^{-\frac{1}{2} l-\frac{1}{4} n}$ for $r \gg 0$. If we cut $u$ smoothly off to zero outside a $k$-independent neighborhood of $\Gamma$, the construction is complete.

The integer $p_{0}$ in the statement of Theorem I is simply $p+p^{\prime}, \bmod 2$. In the case when $n=1$ and $\nu=0, p_{0} \equiv\left[\frac{1}{2} \mu\right], \bmod 2$ where $\mu$ is the Morse index of $\Gamma$ considered as a geodesic arc with fixed end points $(0,0)$ and $(L, 0)$. For more information about the role of Morse indices in this sort of problem the reader should see [3].

\section{Reflected waves}

In this section we assume $\Gamma$ is a closed ray path which is reflected off $\partial M$ at a finite number of points. More precisely, $\Gamma=\bigcup_{i=1} \Gamma_{i}$ where $\Gamma_{i}$ is the 
geodesic arc which is the projection of the curve $\left(x^{i}(t), \xi^{i}(t)\right), t \in\left[t_{i}, t_{i+1}\right]$ in the characteristic flow with $p\left(x^{i}(t), \xi^{i}(t)\right)=1$. We assume

(i) $x^{i}(t) \in M$, for $t \in\left(t_{i}, t_{i+1}\right), x^{i}\left(t_{i}\right)$ and $x^{i}\left(t_{i+1}\right)$ are on $\partial M$, and $x^{i}\left(t_{i}\right)=$ $x^{i-1}\left(t_{i}\right), i=2, \cdots, m$,

(ii) $x^{m}\left(t_{m+1}\right)=x^{1}\left(t_{1}\right)$.

In view of (i) and (ii) we set $x^{i}\left(t_{i}\right)=x^{i}, \xi^{i}\left(t_{i}\right)=\xi_{-}^{i}, \xi^{i}\left(t_{i+1}\right)=\xi_{+}^{i}$ and adopt the convention that indices are reduced $\bmod m$ where necessary. We assume further

(iii) $\xi_{-}^{i}-\xi_{+}^{i-1}$ is normal to $\partial M$ at $x^{i}$, i.e., it annihilates $T_{x^{i}}(M)$,

(iv) $\xi_{-}^{i}-\xi_{+}^{i-1} \neq 0$.

(iii) is the reflection condition, and (iv) implies $\Gamma$ never touches $\Gamma$ tangentially.

Assuming a stability condition analogous to that used previously, we will sketch the construction of a sequence of approximate eigenfunctions concentrated near $\Gamma$, satisfying

$$
\left\|\left(\Delta+k_{m}^{2}\right) u_{m}\right\|=O\left(k^{\frac{1}{2}-\frac{1}{4} n}\right), \quad\left\|u_{m}\right\|>c k_{m}^{-n / 4},
$$

and $u_{m}=0$ on $\partial M$. This sequence will correspond to the "fundamental" sequence in the previous construction, i.e., the sequence with $\alpha=0$. For simplicity we assume $M$ is orientable.

To construct an analogue of the Poincaré map for $\Gamma$ we choose functions $\rho_{i}$ defined near $x^{i}$ such that

(i) $\rho_{i}=0$ on $\partial M$,

(ii) $p\left(x, d \rho_{i}\right)=1$.

Then in ordinary geometrical optics a ray hitting $\partial M$ near $x^{i}$ with data $\left(x, \xi_{+}\right)$ will be reflected to a ray with date $\left(x, \xi_{-}\right)$, where $x \in \partial M$ and

$$
\xi_{-}=\xi_{+}-\left(\xi_{+} \cdot \frac{\partial p}{\partial \xi}\left(x, d \rho_{i}\right)\right) d \rho_{i} .
$$

Hence, considering the induced map on the tangent space to $T^{*}(M)$, we define "reflection" maps

$$
\begin{aligned}
R_{i}: \Lambda_{+}^{i}=\left\{(\delta x, \delta \xi) \in T_{\left(x^{i}, \xi_{+}^{i-1}\right)}\left(T^{*}(M)\right): d \rho_{i} \cdot \delta x=0\right\} & \\
\rightarrow \Lambda_{-}^{i}= & \left\{(\delta x, \delta \xi) \in T_{\left(x^{i}, \xi_{-}^{i}\right)}\left(T^{*}(M)\right): d \rho_{i} \cdot \delta x=0\right\} \\
R_{i}:(\delta x, \delta \xi) \rightarrow(\delta x, \delta \xi)- & \left(0,\left(\left.\delta \xi \cdot \frac{\partial p}{\partial \xi}\left(x, d \rho_{i}\right) d \rho_{i}\right|_{x=x^{i}}\right)\right) \\
& -\left(0,\left.d_{x}\left(\xi_{+}^{i-1} \cdot \frac{\partial p}{\partial \xi}\left(x, d \rho_{i}\right) d \rho_{i}\right)\right|_{x=x^{i}} \cdot \delta x\right) .
\end{aligned}
$$

In defining $R_{i}$ we have made use of the natural identification of $\Lambda_{+}^{i}$ and $\Lambda_{-}^{i}$. A short computation shows $R_{i}$ is a real symplectic map of $\Lambda_{+}^{i}$ to $\Lambda_{-}^{i}$.

Along each arc $\left(x^{i}(t), \xi^{i}(t)\right), t \in\left[t_{i}, t_{i+1}\right]$, we can introduce the flow $\Phi_{i}$ and define $P_{i}$ to be the real symplectic map of 


$$
J_{-}^{i}=\left\{v \in T_{\left(x^{i}, \xi_{-}^{i}\right)}\left(T^{*}(M)\right): \sigma(v, t)=0, \forall t \in T_{\left(x^{i}, \xi_{-}^{i}\right)}\left(C_{i}\right)\right\}
$$

to

$$
J_{+}^{i}=\left\{v \in T_{\left(x^{i+1}, \xi_{+}^{i}\right)}\left(T^{*}(M)\right): \sigma(v, t)=0, \forall t \in T_{\left(x^{i+1}, \xi_{+}^{i}\right)}(C)\right\}
$$

obtained from $\Phi_{i}$ as in $\S 1$. Here $C_{i}=\left\{\left(x^{i}(t), c \xi^{i}(t)\right): c \in \boldsymbol{R}_{+}, t \in\left[t_{i}, t_{i+1}\right]\right\}$.

To construct a Poincaré map we must redefine $R_{i}$ so that it maps $J_{+}^{i-1}$ onto $J_{-}^{i}$. To do this we identify $v \in J_{+}^{i-1}$ and $v^{\prime} \in \Lambda_{+}^{i}$ if $v-v^{\prime} \in T_{\left(x^{i}, \xi_{+}^{i-1}\right)}\left(C_{i-1}\right)$, and identify $w \in J_{-}^{i}$ and $w^{\prime} \in \Lambda_{-}^{i}$ if $w-w^{\prime} \in T_{\left(x^{i}, \xi_{-}^{i}\right)}\left(C_{i}\right)$. Since $R_{i}$ maps $T_{\left(x^{i}, \xi_{+}^{i-1}\right)}\left(C_{i-1}\right) \cap \Lambda_{+}^{i}$ onto $T_{\left(x^{i}, \xi_{-}^{i}\right)}\left(C_{i}\right) \cap \Lambda_{-}^{i}$, with the preceding identifications $R_{i}$ becomes a well-defined real symplectic map of $J_{+}^{i-1}$ onto $J_{-}^{i}$. Hence we can finally define the Poincaré map $P: J_{-}^{1} \rightarrow J_{-}^{1}$ by

$$
P=R_{1} P_{m} \cdots P_{2} R_{2} P_{1} .
$$

$P$ is a real symplectic map. If $P$ has distinct eigenvalues $\lambda_{1}, \bar{\lambda}_{1}, \cdots, \lambda_{n}, \bar{\lambda}_{n}$, we can carry out the construction of $\S \S 1$ and 2 , getting phase functions $\psi_{i}, i=$ $1, \cdots, m$, where $\operatorname{Im} \psi_{i}>0$ off $\Gamma_{i}$ and $\psi_{i}$ satisfies the eichonal equation $\underline{c}_{0}=0$ to second order on $\Gamma_{i}$. Adding constants to the $\psi_{i}$, one can arrange $\psi_{i}\left(x^{i}\right)=$ $\psi_{i-1}\left(x^{i}\right), i=2, \cdots, m$. Then $\psi_{m}\left(x^{1}\right)=\psi_{1}\left(x^{1}\right)+L$, where $L$ is the length of $\Gamma$. The reflections $R_{i}$ were defined so that $d \psi_{i}=d \psi_{i-1}-\left(d \psi_{i-1} \cdot \frac{\partial p}{\partial \xi}\left(x, d \rho_{i}\right)\right) d \rho_{i}$ on $\partial M$ to second order at $x^{i}$. Since $d \rho_{i}$ vanishes on the tangent space to $\partial M$, it follows that $\psi_{i}-\psi_{i-1}$ restricted to $\partial M$ vanishes to third order at $x^{i}$ for $i=2, \cdots, m$, and $\psi_{m}-\psi_{1}-L$ restricted to $\partial M$ vanishes to third order at $x^{1}$.

On each curve $\Gamma_{i}$ the construction of $\psi_{i}$ also yields a matrix $Z_{i}$ analogous to $Z$ in $\S 3$, and we build $u$ as

$$
\begin{aligned}
u= & e^{i k \psi_{1}}\left(\operatorname{det} Z_{1}\right)^{-\frac{1}{2}}-e^{i k \psi_{2}}\left(\operatorname{det} Z_{2}\right)^{-\frac{1}{2}} \\
& +\cdots+(-1)^{m+1} e^{i k \psi_{m}}\left(\operatorname{det} Z_{m}\right)^{-\frac{1}{2}} .
\end{aligned}
$$

The $Z_{i}$ satisfy $\operatorname{det} Z_{i}=\operatorname{det} Z_{i-1}$ at $x^{i}, i=2, \cdots, m$. The eigenvalue condition is imposed by requiring $u\left(x^{1}\right)=0$. This leads to $k=k_{r}$ exactly as in (3.8) with $\alpha=0$ except that $\nu$ is defined by $\nu=0$ if $m$ is even, and $\nu=2$ if $m$ is odd.

Since $u\left(x^{i}\right)=0$ and $\psi_{i}-\psi_{i-1}$ vanishes on $\partial M$ to third order at $x^{i}$, we can modify $u$ near $x^{i}$ so that $u=0$ on $\partial M$ and still maintain $\left\|\left(\Delta+k_{r}^{2}\right) u\right\|=$ $O\left(k_{r}^{(-n+2) / 4}\right)$. As in $\S 3$, cutting $u$ off to zerooutside a $k$-independent neighborhood of $\Gamma$ completes the construction.

In the case of "bouncing ball" waves, i.e., when $\Gamma$ consists of two copies of the same geodesic arc traced in opposite directions, there is an interesting simplification in (4.1). In this case the spaces $J_{i-1}^{+}$and $J_{i}^{-}$may be identified and 
we call the resulting space $J_{i}, i=1,2$. The reflection map $R_{i}$ becomes a map of $J_{i}$ onto $J_{i}$. We introduce coordinates $\left(x_{0}, x^{\prime}\right)$ near $\Gamma$ like those used in $\S 2$ with $x^{1} \sim(0,0)$ and $x^{2} \sim\left(\frac{1}{2} L, 0\right)$, and let $\left(\varphi_{i}\left(x_{0}\right), \dot{\varphi}_{i}\left(x_{0}\right)\right), i=1, \cdots, n$, be the solution of (2.1) with data at $x_{0}=0$ equal to an eigenvector of $P$. As in $\S 2$ this eigenvector is chosen so that $\varphi_{i} \cdot \dot{\bar{\varphi}}_{i}-\dot{\varphi}_{i} \cdot \bar{\varphi}_{i}=-2 \sqrt{-1}$. Let $\left(\eta_{i}\left(x_{0}\right), \dot{\eta}_{i}\left(x_{0}\right)\right)$, $i=1, \cdots, n$, be the solution of $(2.1)$ such that $R_{1}\left(\left(\eta_{i}(0),-\dot{\eta}_{i}(0)\right)\right)=\left(\left(\varphi_{i}(0)\right.\right.$, $\dot{\varphi}(0))$.

Let $T$ be the map $(\delta x, \delta \xi) \rightarrow(\delta x,-\delta \xi)$. The maps $T R_{i}, i=1,2$, are involutions. By the definition of $P$

$$
R_{2}\left(\left(\varphi_{i}\left(\frac{1}{2} L\right), \dot{\varphi}_{i}\left(\frac{1}{2} L\right)\right)=\lambda_{i}\left(\eta_{i}\left(\frac{1}{2} L\right),-\dot{\eta}_{i}\left(\frac{1}{2} L\right)\right)\right.
$$

and, since $T R_{2}$ is an involution and $\left|\lambda_{i}\right|=1$,

$$
\bar{\lambda}_{i}\left(\varphi_{i}\left(\frac{1}{2} L\right), \dot{\varphi}_{i}\left(\frac{1}{2} L\right)\right)=R_{2}\left(\eta_{i}\left(\frac{1}{2} L\right), \dot{\eta}_{i}\left(\frac{1}{2} L\right)\right) .
$$

Imitating the procedure in $\S 2$,

$$
\varphi_{i} \cdot \dot{\eta}_{j}-\dot{\varphi}_{i} \cdot \eta_{j}=e_{i j}, \quad \bar{\varphi}_{i} \cdot \dot{\eta}_{j}-\dot{\bar{\varphi}}_{i} \cdot \eta_{j}=f_{i j} \cdot
$$

As before $e_{i j}$ and $f_{i j}$ are constants, and since $R_{2}$ is a real symplectic map, $e_{i j}=\lambda_{i} \bar{\lambda}_{j} e_{i j}$ and $f_{i j}=\bar{\lambda}_{i} \bar{\lambda}_{j} f_{i j}$. Hence $e_{i j}=0$ for $i \neq j$ and $f_{i j} \equiv 0$. Since we also have (see $\S 2$ )

$$
\begin{aligned}
& \varphi_{i} \cdot \dot{\bar{\varphi}}_{j}-\dot{\varphi}_{i} \cdot \bar{\varphi}_{j}=0, \quad i \neq j, \\
& \varphi_{i} \cdot \dot{\varphi}_{j}-\dot{\varphi}_{i} \cdot \varphi_{j} \equiv 0,
\end{aligned}
$$

and $\left\{\left(\varphi_{i}, \dot{\varphi}_{i}\right)\right\}_{i=1}^{n} \cup\left\{\bar{\varphi}_{i}, \dot{\bar{\varphi}}_{i}\right\}_{i=1}^{n}$ forms a basis for $\boldsymbol{C}^{2 n}$, it follows

$$
\left(\eta_{i}, \dot{\eta}_{i}\right)=c_{i}\left(\bar{\varphi}_{i}, \dot{\bar{\varphi}}_{i}\right)
$$

for constants $c_{i}$ determined by $\varphi_{i}(0)=\eta_{i}(0)$. The matrix $B$ derived from the $\eta_{i}$ is defined by $B \eta_{i}=-\dot{\eta}_{i}$.

Continuing with the construction of the approximate eigenfunctions as in $\S 2$, we eventually get

$$
u=e^{i k \psi_{1}}\left(\operatorname{det} Z_{1}\right)^{-\frac{1}{2}}-e^{i k \psi_{2}}\left(\operatorname{det} Z_{2}\right)^{-\frac{1}{2}}
$$

However, the observations of the preceding paragraph imply $\psi_{2}=-\bar{\psi}_{1}$, and for suitable $\beta \in R, e^{i \beta} \operatorname{det} Z_{2}=e^{-i \beta} \operatorname{det} \bar{Z}_{1}$. Thus replacing $u$ by $e^{-\frac{1}{2} i \beta} u$ and modifying $Z_{1}$ appropriately we have

$$
u=w-\bar{w}
$$

where $w=e^{i k \psi_{1}}\left(\operatorname{det} Z_{1}\right)^{-\frac{1}{2}}$. This is consistent with the form of $u$ in the case $n=1$ which was found in [10]. 
The chief implication of (4.2) is that the argument that will be used in $\S 5$ to show there are two eigenvalues of $\Delta$ in an interval about $-k_{r, \alpha}^{2}$ of radius $O\left(k_{r, \alpha}^{\frac{1}{2}}\right)$ fails for bouncing ball waves.

\section{Implications}

For the domain of $\Delta$ we take the subspace of $C_{0}^{\infty}(M \cup \partial M)$ consisting of functions vanishing on $\partial M$. With this domain $\Delta$ is symmetric, densely defined and nonpositive. Thus, by Friedrichs' theorem, (cf. [7, pp. 325-326]), it has a nonpositive self-adjoint extension $\Delta_{M}$. The norm of $\left(\Delta_{M}-\lambda I\right)^{-1}$ equals the distance from $\lambda$ to the spectrum of $\Delta_{M}$ (cf. [7, p. 272]).

If $M \cup \partial M$ is compact, then $\Delta_{M}$ is the graph closure of $\Delta$ and has pure point spectrum. Since the $u_{r, \alpha}$ constructed in $\S \S 3$ and 4 satisfy

$$
\begin{gathered}
\left\|\left(\Delta+k_{r, \alpha}^{2}\right) u_{r, \alpha}\right\|=O\left(k_{r, \alpha}^{-n / 4-|\alpha| / 2+1 / 2}\right), \\
\left\|u_{r, \alpha}\right\| \geq c_{\alpha} k_{r, \alpha}^{-n / 4-|\alpha| / 2},
\end{gathered}
$$

it follows the distance from $k_{r, \alpha}^{2}$ to an eigenvalue of $\Delta_{M}$ is $O\left(k_{r, \alpha}^{1 / 2}\right)$.

In the case where $\Gamma$ is a closed geodesic, as in $\S \S 1-3$, we claim that there are at least two eigenvalues of $\Delta_{M}$, counted by multiplicity, whose distance to $k_{r, \alpha}^{2}$ is $O\left(k_{r, \alpha}^{1 / 2}\right)$. To see this consider

$$
\left(u_{r, \alpha}, \bar{u}_{r, \alpha}\right)=\int_{M} u_{r, \alpha}^{2} d x=\int d x^{\prime} \int_{0}^{L} e^{i 2 k_{r, \alpha} x_{0}}\left(e^{-i 2 k_{r, \alpha} x_{0}} u_{r, \alpha}^{2}\right) d x_{0} .
$$

Integration by parts once in $x_{0}$ shows $\left(u_{r, \alpha}, \bar{u}_{r, \alpha}\right)=O\left(k_{r, \alpha}^{-n / 2-|\alpha|-1}\right)$. Thus we have

$$
\begin{aligned}
\|\left(\Delta+k_{r, \alpha}^{2}\right) \frac{u_{r}}{\left\|u_{r}\right\|} & =O\left(k_{r, \alpha}^{1 / 2}\right), \\
\|\left(\Delta+k_{r, \alpha}^{2}\right) \frac{\bar{u}_{r}}{\left\|\bar{u}_{r}\right\|}=O\left(k_{r, \alpha}^{1 / 2}\right), & \\
\left(\frac{u_{r, \alpha}}{\left\|u_{r, \alpha}\right\|}, \frac{\bar{u}_{r, \alpha}}{\left\|\bar{u}_{r, \alpha}\right\|}\right) & =O\left(k_{r, \alpha}^{-1}\right) .
\end{aligned}
$$

Now an elementary argument, which we leave to the reader, gives the desired result.

Suppose $\Delta_{M}$ has only two eigenvalues in an interval about $-k_{r, \alpha}^{2}$ of length $d_{r}$, where $\lim _{r \rightarrow \infty}\left(k_{r, \alpha}^{1 / 2}\right) / d_{r}=0$. Let $P_{r}$ denote the orthogonal projection on the subspace spanned by $u_{r, \alpha}$, and let $\tilde{P}_{r}$ denote the spectral projection for $\Delta_{M}$ on the interval $\left[-d_{r}-k_{r, \alpha}^{2},-k_{r, \alpha}^{2}+d_{r}\right]$. Then one can show $\lim _{r \rightarrow \infty}\left\|P_{r}-\tilde{P}_{r}\right\|$ $=0$.

However, Arnold has given an example where none of the $u_{r, \alpha}$ 's are close to true eigenfunctions (see [1]). We offer the following argument that, in a practical sense, it is impossible to distinguish the $u_{r, \alpha}$ 's from true eigenfunctions 
when $r$ is sufficiently large. For functions $u(x, t)$ on $M \times R$ we introduce the energy

$$
E(u)=\int_{M}\left(\sum g^{i j} \frac{\partial u}{\partial x_{i}} \frac{\partial \bar{u}}{\partial x_{j}}+\left|\frac{\partial u}{\partial t}\right|^{2}\right) d \omega
$$

where $d \omega$ is the volume form on $M$. Then, if $u$ is a solution to $\partial^{2} u / \partial t^{2}-\Delta u$ $=0, E(u)$ is independent of $t$.

Let $u(x, t)$ be the solution to the mixed problem: $\partial^{2} u / \partial t^{2}-\Delta u=0, u(x, 0)$ $=u_{r, \alpha}(x),(\partial u / \partial t)(x, 0)=i k_{r, \alpha} u_{r, \alpha}(x)$ and $u(x, t)=0$ on $\partial M$. A simple estimate from Duhamel's formula (cf. [7, pp. 486-487]) shows $E\left(u(x, t)-e^{i k_{r}, \alpha t} u_{r, \alpha}\right)$ $=O\left(k_{r, \alpha}^{-(n / 2+|\alpha|-1)}|t|\right)$. One checks easily $E(u(x, t))=E(u(x, 0)) \geq c_{\alpha} k_{r, \alpha}^{-(n / 2+|\alpha|-3)}$. Hence, given $T$ and $\varepsilon$, there is an $r_{0}$ such that for $r \geq r_{0}$, the standing wave $v(x, t)=c_{r} e^{i k_{r}, \alpha t} u_{r, \alpha}(x)$ differs from a true solution to $\partial^{2} u / \partial t^{2}-\Delta u=0$ by $\varepsilon$ in energy norm for $|t|<T$. The constant $c_{r}$ is chosen so that $E(v(x, 0))$ $=1$. Note that this argument applies equally well when $M \cup \partial M$ is not compact.

\section{References}

[1] V. I. Arnol'd, Modes and quasimodes, Functional Anal. Appl. 6 (1972) 94-101.

[2] V. M. Babich \& V. F. Lazutkin, Eigenfunction concentrated near a closed geodesic, Topics in Math. Phys. Vol. 2 (M. S. Birman, ed.), Consultant's Bureau, New York, 1968, 9-18.

[ 3 ] J. J. Duistermaat, On the Morse index in variational calculus, Advances in Math. 21 (1976) 173-195.

[4] V. Guillemin, Symplectic spinors and partial differential equations, Sympos. Symplectic Geometry and Mathematical Phys., Centre Nat. Recherche Sci., Aix-en-Provence, 1974.

[5] V. Guillemin \& A. Weinstein, Eigenvalues associated with a closed geodesic, Bull. Amer. Math. Soc. 82 (1976) 92-94, Correction and addendum, Bull. Amer. Math. 82 (1976) 966.

[6] L. Hörmander, On the existence and regularity of solutions of linear pseudodifferential equations, Enseignement Math. 17 (1971) 99-163.

[ 7 ] T. Kato, Perturbation of linear operators, Springer, New York, 1966.

[ 8 ] J. B. Keller \& S. I. Rubinow, Asymptotic solution of eigenvalue problems, Ann. Physics 9 (1960) 24-75.

[9] V. F. Lazutkin, Construction of an asymptotic series of eigenfunctions of the bouncing ball type, Proc. Steklov Inst. Math. 95 (1968) 106-118.

[10] R. Smith, Bouncing ball waves, SIAM J. Appl. Math. 20 (1974) 5-14. 